\title{
Synthesis and Electroluminescence Studies of Manganese Doped Cadmium Sulfide Nanoparticles
}

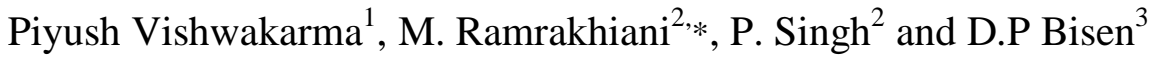 \\ ${ }^{1}$ Lingaya's University, Faridabad, India \\ ${ }^{2}$ Rani Durgawati University, Jabalpur, India \\ ${ }^{3}$ Pt. Ravi Shanker Shukla University, Raipur, India
}

\begin{abstract}
The nanoparticles of cadmium sulfide (CdS) doped with various concentration of manganese (Mn) were synthesized by wet chemical route and characterized by TEM and XRD techniques. The average particle size found by TEM is $4.256 \mathrm{~nm}$ and XRD gives regularity of atoms up to about $2 \mathrm{~nm}$ only. The absorption spectra show absorption edge at $448 \mathrm{~nm}$ for all the samples. From the increased effective band gap as $2.76 \mathrm{eV}$, the particle size was estimated by theory and is found to be nearly same as that obtained by TEM.

The Voltage dependence of electroluminescence (EL) brightness of the CdS:Mn shows exponential increase. The linear voltage-current characteristic indicates ohmic nature. The EL brightness at a particular voltage is found to increase by increasing Mn doping up to $1.0 \%$, but for higher percentage of Mn the EL brightness is reduced. It is also seen that Mn does not influence the threshold voltage.
\end{abstract}

Keyword: CdS: Mn nanoparticle, XRD, TEM, Absorption spectra, Electroluminescence.

\section{INTRODUCTION}

Nanoparticles are generally categorized as the class of materials that fall between the molecules and bulk solids, with an average size between 1 to $50 \mathrm{~nm}$. Nanoparticles exhibit physical and chemical properties different from either the individual molecules or the extended solid, hence attracting an enormous attention during the past few decades [1-8]. The change in the property of nanoparticles are driven mainly by two factors namely the increase in the surface area to volume ratio and change in the electronic structure of the material due to the quantum mechanical effects with decreasing particle size.

Quantum dots or semiconductor nanoparticles, whose size is comparable or smaller than Bohr diameter of exciton, are finding increasing applications. These have much higher quantum efficiency for fluorescence as compared to their bulk counterparts. Their fluorescence wavelength can be easily changed by changing the particle size or by doping them with suitable ions. Surface charges as well as presence of capping molecules can modify the fluorescence emission of nanoparticles. Surface functionalized nanoparticles can be attached to larger particles or modified so as to suite a particular environment or application.

Apart from the effect of a large surface area, the optical and electronic properties of the materials also undergo drastic changes as a function of the size blow a certain limit.

*Address correspondence to this author at the Rani Durgawati University, Jabalpur, India; Tel; 09826353264;E-mail: mramrakhiani@hotmail.com, drpranav_singh@yahoo.co.nz
At sizes comparable to or less than exciton Bohr radius, the exciton binding energy and the oscillator strength increases due to

the enhanced overlap between the electron and hole wave function [9].

The emission properties can further be tuned by incorporating impurity atoms into the pure semiconductor lattice also known as doping; this process provides new states in the band gap region of the semiconductor. These doped nanoparticles provide an alternate path way for recombination of the electron-hole pair, thus gives rise to an emission of different energy, from the host semiconductor. Doped semiconductor nanoparticles are being extensively investigated to obtain basic information on impurity states in quantum dots and to examine their potential application in novel light emitting devices [1].

Present paper reposts the light emission from cadmium sulfide nanoparticles, doped with various concentration of manganese, by application of A.C. voltage.

\section{EXPERIMENTAL WORK}

\subsection{Preparation of CdS Nanoparticles}

In the present work, CdS nanoparticles have been prepared using wet chemical route [10]. Dilute solutions of cadmium chloride $\left(\mathrm{CdCl}_{2}-0.01 \mathrm{M}\right.$ concentration) and sodium sulfide $\left(\mathrm{Na}_{2} \mathrm{~S}-0.01 \mathrm{M}\right.$ concentration) were mixed in presence of capping agent merceptoethenol (0.01 M $\left.\mathrm{C}_{2} \mathrm{H}_{5} \mathrm{OSH}\right)$. Solution of manganese chloride $\left(\mathrm{MnCl}_{2}-0.01 \mathrm{M}\right.$ 


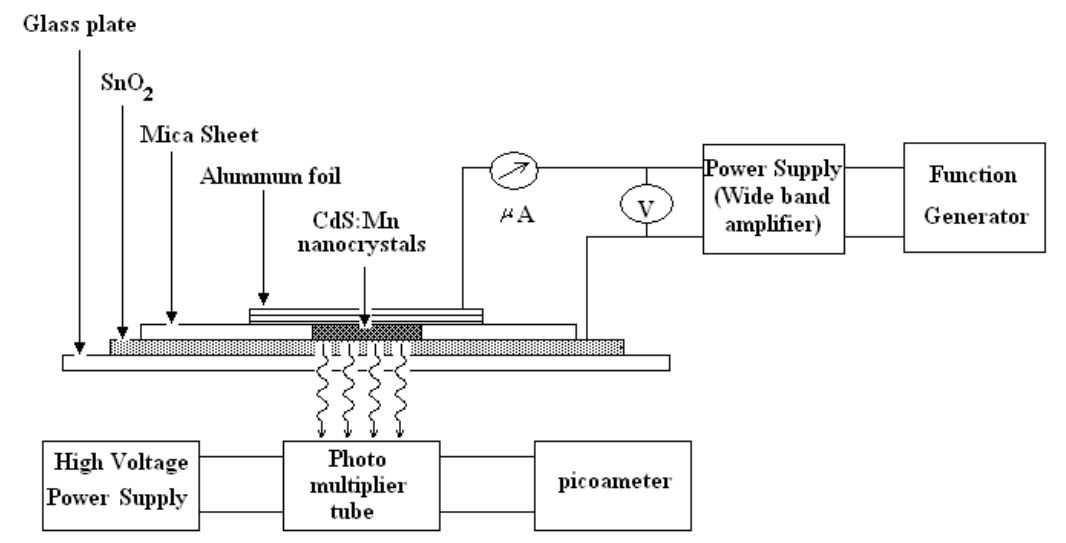

Fig. (1). Set up for EL measurement.

concentration) was added to cadmium chloride solution in proper amount for doping.

The aqueous solution of merceptoethenol was added drop-wise in the solution of $\mathrm{CdCl}_{2}$ (and $\mathrm{MnCl}_{2}$ in proper ratio) with the help of a burette at the rate of $1 \mathrm{ml}$ per minute, stirring the solution continuously. A magnetic stirrer was used for stirring the solution. There after $0.01 \mathrm{M}$ solution of $\mathrm{Na}_{2} \mathrm{~S}$ was mixed drop-wise into the solution. Subsequently yellow color solution obtained was kept for 24 hours. Yellow precipitate settled down in the bottom of the flask. This precipitate was collected and washed several times with the double distilled water. The reaction can be summarized in the equation as

$\mathrm{CdCl}_{2}+\mathrm{Na}_{2} \mathrm{~S}=\mathrm{CdS}+2 \mathrm{NaCl}$

This washed precipitate was centrifuged for 30 minute at $3500 \mathrm{rpm}$ with the help of a centrifuge for the separation of the nanoparticle. Finally the precipitate was spread over a glass substrate and air dried at room temperature. The capping agent $\mathrm{C}_{2} \mathrm{H}_{5} \mathrm{OSH}$ controls the particle size.

A number of samples were prepared by using different concentration of manganese varying from $0.0 \%$ to $5 \%$.

\subsection{Characterization of Samples}

A number of experimental techniques are employed to yield structural information of nanocrystalline materials. All techniques are complimentary to each other. In present investigations CdS:Mn nanocrystals are characterized by Transmission electron microscopy (TEM) and X-Ray diffraction (XRD) techniques. TEM of CdS:Mn nanocrystals were done by using Techani $20 \mathrm{G}$ electron microscope. The XRD was done by Rigaku Rotating Anode (H-3R) diffractometer with irradiation from $\mathrm{K}_{\alpha}$ line of copper $(\lambda=1.5418 \AA)$.

Optical absorption spectra of CdS nanoparticles were recorded by using Perkin Elemer Lambda-12 spectrometer in the UV/VIS region ranging from $700 \mathrm{~nm}$ to $400 \mathrm{~nm}$.

\subsection{Preparation of Electroluminescence (EL) Cell}

For the study of electroluminescence (EL) of samples, the EL cell was prepared by a triple layer structure namely a nanocrystals emission layer, sandwiched between two electrodes. One of the electrodes has to be transparent for the

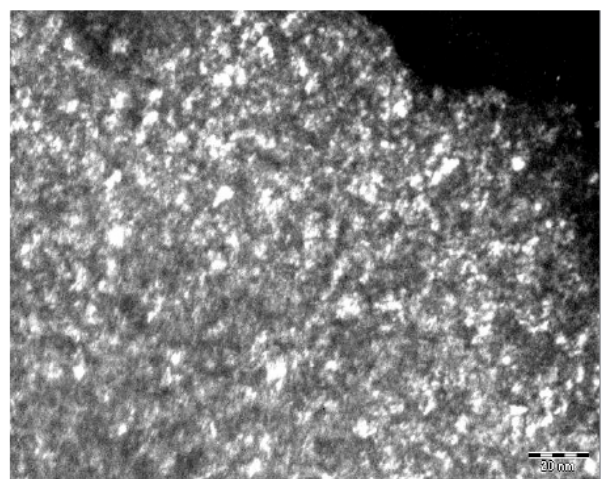

Fig. (2). Transmission electron microscopy images of CdS: Mn $(0.3 \%)$.

generated light to come out. The transparent electrode has been prepared by deposition of thin film of $\mathrm{SnO}_{2}$ by chemical vapor deposition on clean glass plate. The glass plates with resistance less then $100 \mathrm{ohms} / \mathrm{cm}$ were used in the investigation.

El cell was prepared by placing a mica sheet, having a small window, over the $\mathrm{SnO}_{2}$ coated conducting transparent glass plate. This $\mathrm{SnO}_{2}$ coated glass behaves as first electrode. Nanoparticle of CdS or CdS:Mn were first mixed in DMSO (di methyle sulfo oxide) and then fixed inside the window and aluminum foil was pressed tightly, which behaves as second electrode. The layer of nanoparticle should be uniform and thin. The A.C. electric field is applied between these two electrodes.

\subsection{EL Measurements}

The arrangement for the measurement of EL was shown in Fig. (1). The EL brightness was measured by photomultiplier tube (PMT) operated at high voltage and output fed to a picoammeter. For the EL studies, a particular frequency is set in the audio generator and voltage applied to the EL cell was increased from 0 to 700 volts and corresponding current and EL brightness were recorded. The EL was studied at various frequencies for $\mathrm{CdS}$ nanoparticles with different $\mathrm{Mn}$ concentrations.

\section{RESULTS AND DISCUSSION}




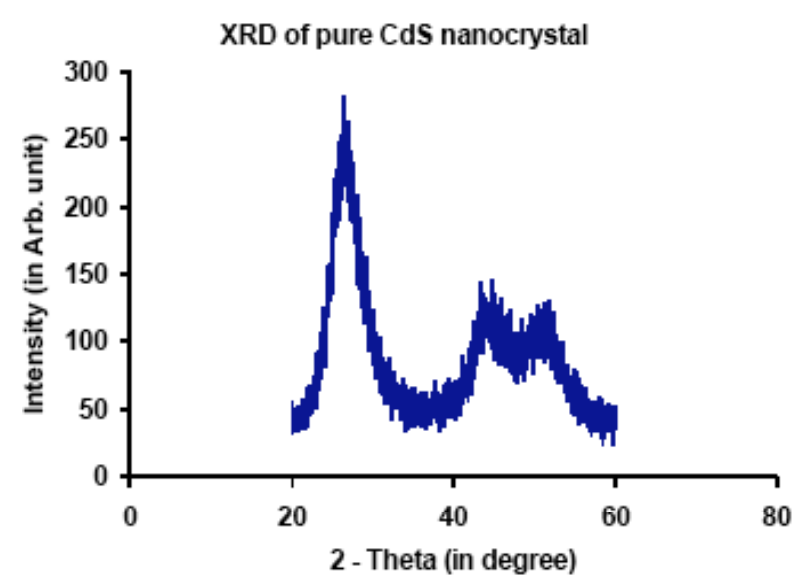

(a)

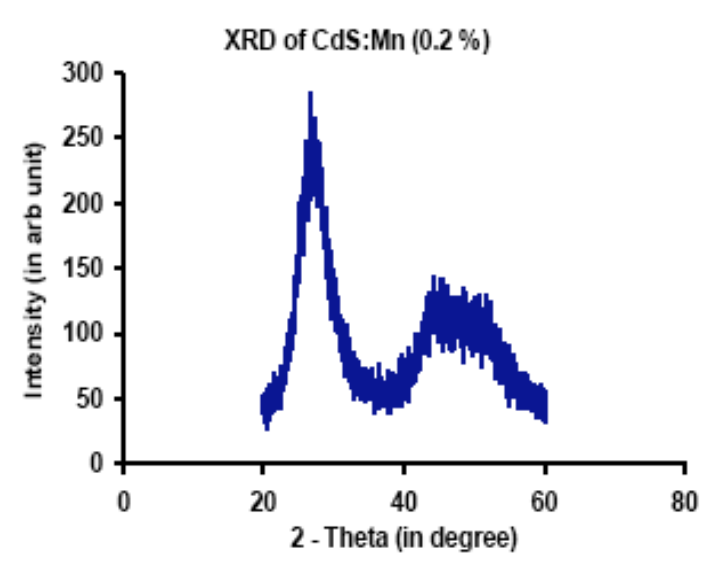

(b)

Fig. (3). X-Ray Diffraction pattern of CdS:Mn nanoparticle.

A typical TEM image of CdS:Mn $(0.3 \% \mathrm{Mn})$ is shown in Fig. (2). The average particle size of CdS:Mn has been obtained as $4 \mathrm{~nm}$.

Fig. (3) shows the XRD pattern of CdS:Mn nanocrystals. The analysis of XRD reveals that CdS:Mn nanocrystals have zinc-blende crystal structure. For all the samples three peaks are observed corresponding to diffraction from (111), (220) and (311) planes. For lower concentration of $\mathrm{Mn}$, three peaks

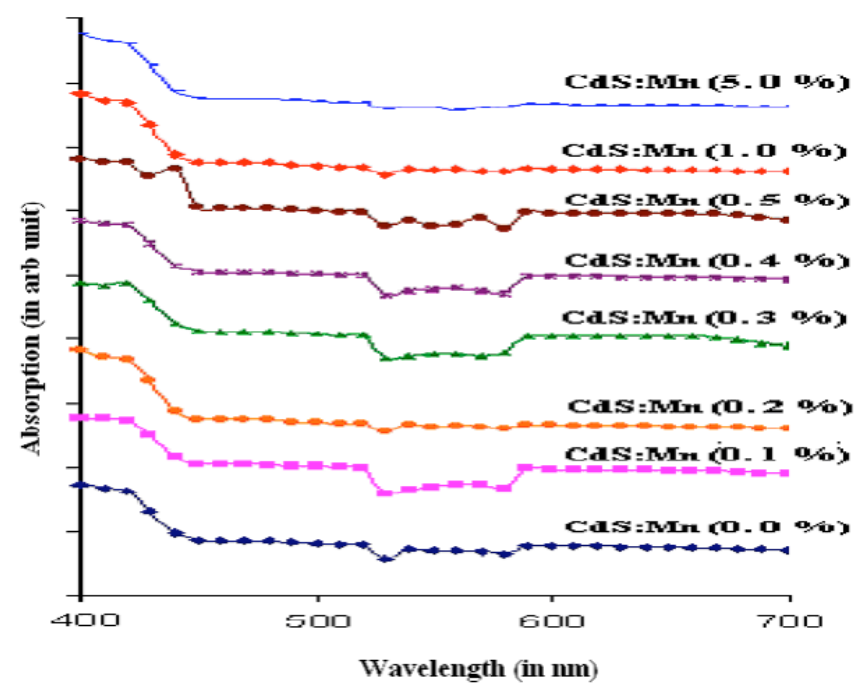

Fig. (4). Absorption spectra of CdS:Mn nanoparticle.

are seen separately, but as the Mn concentration increases second and third peaks corresponding to (220) and (311) planes get merged.

Due to the size effect, the XRD peak tends to broaden and their width increases as the size of crystals decreases. The crystalline size has been estimated from the broadening of the first diffraction peak using Debye-Scherrer formula [11]:

$R=\frac{K \lambda}{\beta \operatorname{Cos} \theta}$ where $\mathrm{R}$ is crystallite size, $\mathrm{K}$ is constant, $\theta$ is Bragg angle, $\lambda$ is wave length and $\beta$ is Full-Width at Half-Maximum (FWHM) of peak. The average particle size of CdS:Mn nanocrystals has been obtained $2 \mathrm{~nm}$. XRD gives the regularity in the atomic arrangement in the samples where as TEM gives the full size of the particle including non-crystalline part also.

\subsection{Absorption Spectra}

The optical absorption spectra of CdS:Mn nanocrystals ware recorded for all the samples having different percent of Mn impurity from $0.0 \%$ to $5.0 \%$ and shown in Fig. (4). The absorption edge is found for all the samples at $448 \mathrm{~nm}$. The effective band gap $\left(E_{g}\right)$ is calculated as $2.76 \mathrm{eV}$. From optical absorption spectra it is found that Mn concentration does not influence to the absorption spectra. It is known that as the particle size decreases there is increase in effective band gap of semiconductors due to confinement of charge carriers in a very small dimension [2] as -

$$
\Delta E=\frac{\hbar^{2} \pi^{2}}{2 r^{2}}\left[\frac{1}{m_{e}^{*}}+\frac{1}{m_{h}^{*}}\right]-\frac{1.786 e^{2}}{\varepsilon r}-\frac{0.124 e^{4}}{\hbar^{2} \varepsilon^{2}}\left[\frac{1}{m_{e}^{*}}+\frac{1}{m_{h}^{*}}\right]^{-1}
$$

where $m_{e}^{*}$ and $m_{h} *$ are the effective masses of electron and hole respectively, $r$ is radius of the particle and $\varepsilon$ is the dielectric constant of the semiconductor. The second and third terms are much smaller than the first term [12], therefore may be neglected and the expression reduces to -

$$
\Delta E=\frac{\hbar^{2} \pi^{2}}{2 r^{2}}\left[\frac{1}{m_{e}^{*}}+\frac{1}{m_{h}^{*}}\right]
$$

The values of the effective mass of electrons and holes for CdS are $m_{e}=0.19 m_{o}$ and $m_{h}=0.8 m_{o} ; m_{o}$ being the electron mass. Substituting all the values and rearranging we get

$$
r=\frac{1.5606}{\Delta E^{1 / 2}}
$$

The effective band gap of the nanocrystalline samples is obtained from the absorption spectra and increase in band 


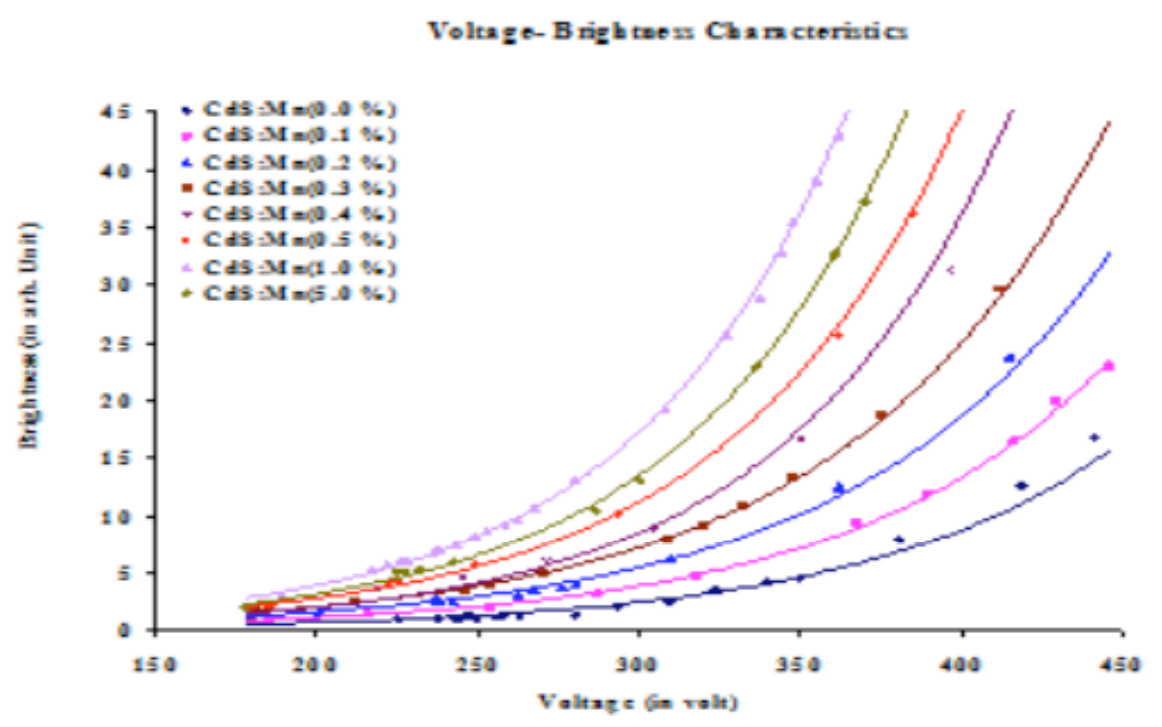

Fig. (5). Voltage - Brightness characteristics of CdS:Mn nanoparticles.

Voltage-current Charecteristics

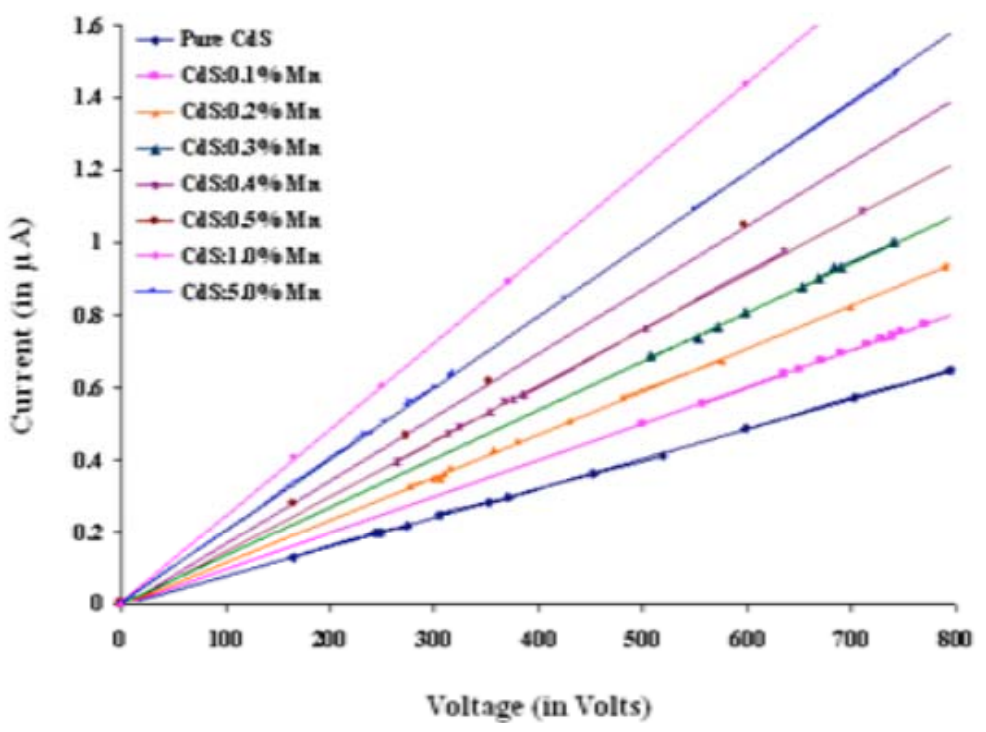

Fig. (6). Voltage current characteristics of CdS:Mn nanoparticles.

gap $(\Delta \mathrm{E})$ is determined by comparing it with the standard band gap of CdS as $2.42 \mathrm{eV}$. The particle size estimated using the above relation is found to be $5.35 \mathrm{~nm}$ for all the samples.

\subsection{Electroluminescence Studies}

The Electroluminescence (EL) was observed in all the samples of CdS:Mn and their brightness have been recorded at various frequencies and voltages and different characteristics have been studied.

\subsubsection{Voltage - Brightness Characteristics}

The voltage dependence of EL brightness (B) of CdS doped with $\mathrm{Mn}(0.0 \%, 0.1 \%, 0.2 \%, 0.3 \%, 0.4 \%, 0.5 \%, 1.0 \%$ and $5.0 \%$ ) at frequency $1 \mathrm{~K} \mathrm{~Hz}$ is shown in Fig. (5). Voltagebrightness curves indicate that as the applied input voltage (V) across the EL cell increases, the emission starts at a particular threshold voltage, and initially increases slowly and then rapidly, giving exponential relation between them. The brightness was observed between 100 volts to 800 volts for all the samples at different frequencies (from $900 \mathrm{~Hz}$ to 1400 $\mathrm{Hz}$ ). At a particular frequency the threshold voltage for all the samples of CdS:Mn is found to be approximately same. The electroluminescent efficiency depends on the carrier lifetime of the injected charge carriers.

\subsubsection{Voltage-Current Characteristics}

The voltage-current characteristic of CdS doped with $\mathrm{Mn}$ $(0.0 \%, 0.1 \%, 0.2 \%, 0.3 \%, 0.4 \%, 0.5 \%, 1.0 \%$ and $5.0 \%)$ at frequency $1 \mathrm{~K}$ is shown in Fig. (6). It is clear from the observation made that as the input voltage increases there is continuous increase in the current. In the present study linear relation is obtained between voltage and current. This indicates the ohmic nature of impedance. The slop of the curve increases on increasing percentage of Mn impurity up to 1.0 $\%$ then decreases at higher $\mathrm{Mn}$ concentration (5.0\%). I-V 
Mn concentration-Impedence Charecteristics

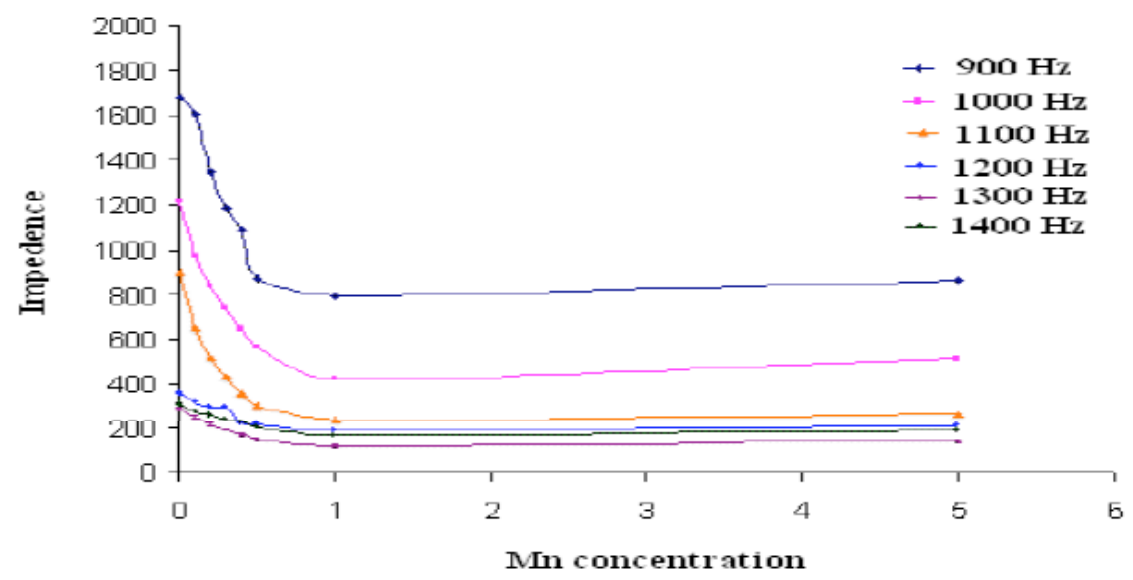

Fig. (7). Variation of impedance with Mn concentration.

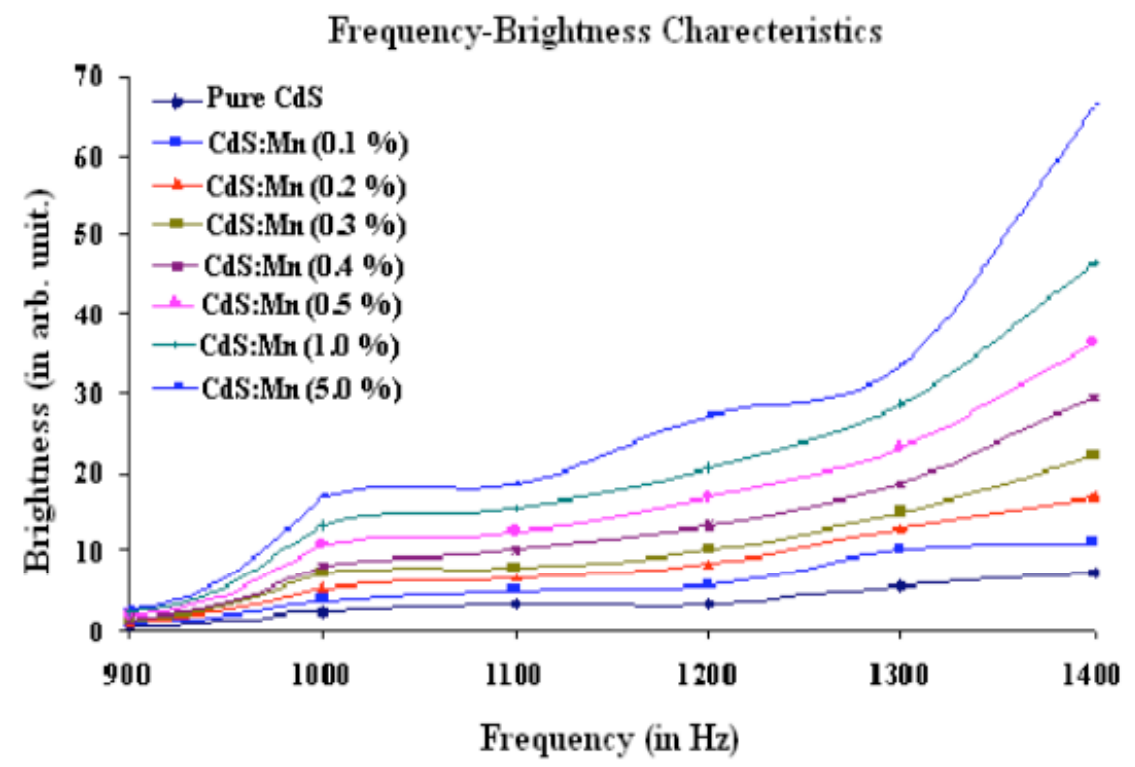

Fig. (8). Variation of brightness with Frequency.

property of the devices depends on the strength of the electric field. In this model, carriers tunnel through barrier at the nanoparticle and electrode interface under the influence of an electric field. The holes tunnel across an energy barrier into the valance band, and electrons tunnels across the conduction band of nanoparticle. These carriers are transported through a series of field associated ionization steps (hopping mechanism).

\subsubsection{Impedance}

The impedance of the EL cells has been determined from the slope of voltage-current characteristic. The impedance is found to depend on the Mn Concentration. The Mn concentration versus impedance curve is shown in Fig. (7). From this figure it is clear that, at a particular frequency, by increasing Mn concentration, impedance decreases up to $1.0 \%$ $\mathrm{Mn}$, then starts increasing for higher Mn \%. By increasing frequency impedance decreases; the decrement behavior is found approximately similar for all the frequencies.

\subsubsection{Effect of Frequency}

The EL of CdS:Mn nanoparticles was observed at different frequencies from $900 \mathrm{~Hz}$ to $1400 \mathrm{~Hz}$ and it is found that on increasing the frequency of the input signal, EL brightness increases (Fig. 8). The increase in EL brightness with frequency can be understood on the basis that, emptying and refilling of EL centers take place more rapidly with increase in frequency.

\subsubsection{Effect of Doping}

Manganese is one of the most important dopent in CdS. It is clear from Fig. (9) that on increasing the percentage of Mn doping, the EL brightness increases up to $1.0 \%$ but for higher percentage of $\mathrm{Mn}$, there is concentration quenching and the EL brightness is reduced. The maximum brightness is observed for the sample of $1.0 \% \mathrm{Mn}$ doping. It is also seen that Mn does not influence the threshold voltage, but it affects only brightness.

Electroluminescence (EL) of nanocrystals is the radiative recombination of electron and hole pairs. The most suited relation for the voltage dependence of EL brightness may be given by [13] 
Concentration-Brightnes s charecte ristic s

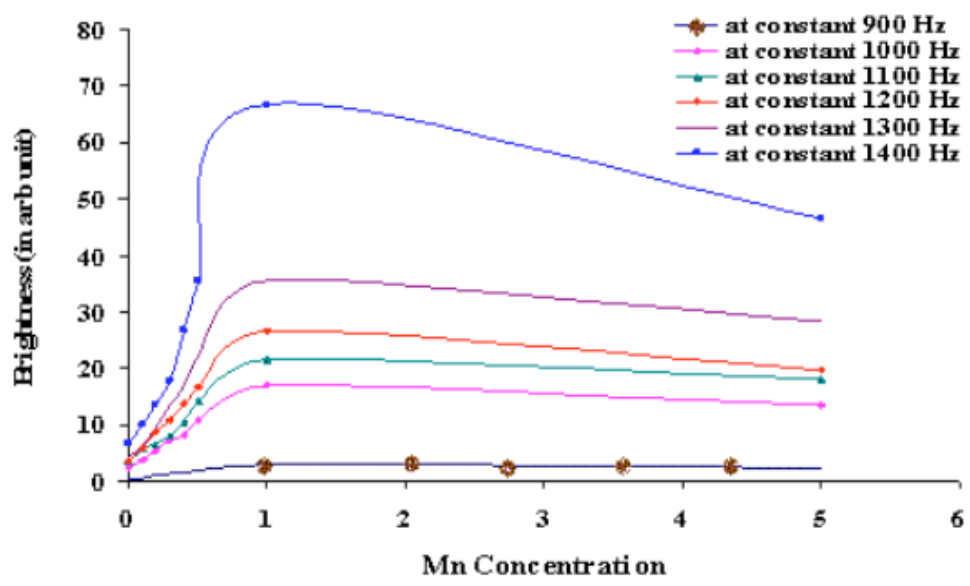

Fig. (9). Variation of brightness with Mn Concentration.

$\mathrm{B}=\mathrm{B}_{0} \exp (-\mathrm{b} / \sqrt{V})$

where $\mathrm{B}_{\mathrm{o}}$ and $\mathrm{b}$ are parameters, which depend on temperature and frequency of the alternating voltage, the material and other details of the construction of EL cell. In an attempt to drive the mechanism of EL excitation, plot between $\log B$ Versus $100 / \mathrm{V}^{1 / 2}$ is considered which is found to be straight line with negative slope. For CdS:Mn nanocrystals, equation (6) successfully explains the voltage-brightness characteristics. The presence of square root of voltage is generally explained on the basis of acceleration collision mechanism [14].

By equation (6) the value of $\mathrm{B}_{0}$ and $\mathrm{b}$ at various frequencies were calculated for different $\mathrm{Mn}$ concentration. It is found that on increasing both frequency and Mn concentration first the value of constant $\mathrm{B}_{0}$ decreases continuously in a systematic manner but for higher frequency and higher Mn concentration, it become high.

As molecules, nanocrystals can interact with electric field via their electronic transitions, but because of their solid state nature, they exhibit different photo-physics. It is generally accepted that luminescent process in semiconductors starts with the initial excitation of an electron into the conduction band by electric field. The emission then occurs via a radiation-less relaxation of electron or hole to a recombination centre followed by radiative recombination [13]. In electroluminescence process electrons from valence band are excited to conduction band by an electric field. These excited electrons can be de-excited to some surface or defect state which cause recombination through non-radiative transition. Other possible path way is the energy transfer to $\mathrm{Mn}^{2+}$ ions in the ${ }^{4} \mathrm{~T}_{1}$ level and the subsequent radiative decay to the ${ }^{6} \mathrm{~A}_{1}$ level [1]. The incorporation of $\mathrm{Mn}^{2+}$ into the $\mathrm{CdS}$ lattice, reduce the probability of non-radiative recombination as a consequence of more efficient ${ }^{4} \mathrm{~T}_{1} \rightarrow{ }^{6} \mathrm{~A}_{1}$ process.

The passivation of surface states can enhance the contribution of radiative recombination in nanomaterials [15]. In case of CdS:Mn, trapping of charge carriers by $\mathrm{Mn}^{2+}$, which is a very fast process, competes with trapping of carriers in surface states. The efficiency of first process over second one is reflected in the ratio of the peak intensity in the lumi- nescence spectra of CdS:Mn nanoparticles. Passivation of surface states reduces the non-radiative recombination rate with the subsequent enhancement in the quantum yield of nanoparticles.

\section{CONCLUSION}

The studies have revealed that the CdS nanoparticles doped with various concentrations of Mn have size of the order of $\sim 4 \mathrm{~nm}$ where as the crystalline $\mathrm{CdS}$ in the particles have only $\sim 2 \mathrm{~nm}$ dimension. Mn doping improves the EL quantum yield up to $1 \% \mathrm{Mn}$, but for higher concentration, the EL brightness is reduced. Electroluminescence (EL) brightness of the CdS:Mn increases exponentially with voltage, while the voltage-current characteristic shows straight line indicating ohmic nature. It is also seen that Mn does not influence the threshold voltage. Relation between voltage and brightness indicate that EL is produced by accelerationcollision mechanism.

\section{CONFLICT OF INTEREST}

None declared.

\section{ACKNOWLEDGMENT}

None declared.

\section{REFERENCES}

[1] Bhargava, R.N.; Gallagher, D.; Welker, T. Doped nanocrystals of semiconductors - a new class of luminescent materials. J. Lumi., 1994, 60, 61, 275-280.

[2] Sahu, S.N.; Nanda, K.K. Nanostructure semiconductors: physics and applications. PINSA, 2001, 67 A, 103-130.

[3] Zhou, H.; Hofmann, D.M.; Hofstaetter, A.; Meyer, B.K. Magnetic resonance investigation of $\mathrm{Mn}^{2+}$ in $\mathrm{ZnO}$ nanocrystals. J. Appl. Phys., 2003, 94(3), 1965-68.

[4] Tan, Y.T.; Kamiya, T.; Durrani, A.K.; Ahmad, H. Room temperature nanocrystalline silicon single-electron transistors. J. Appl. Phy., 2003, 94(1), 633-637.

[5] Sahoo, T.; Tripathi, S. K.; Nandy, S.; Pandey, B. Microstructural and photoluminescence studies on hydrothermally synthesized $\mathrm{Ce}$ doped barium titanate nanocrystals. Mater. Sci. Eng. B., 2006, 131, 277-280.

[6] Sahoo, T.; Pradhan, G.K.; Rath, M.K.; Pandey, B.; Verma, H.C.; Nandy, S.; Chattopadhyay, K.K.; Anand, S. Characterisation and phototluminescence studies on hydrothermally synthesized Mn- 
doped barium titanate nanocrystals. Mater. Lett., 2007, 61, 482123.

[7] Anikeeva, O.P.; Halpert, J.E.; Bawendi, M.G.; Bulovie, V. Quantum dot light-emitting devices with electroluminescence tunable over the entire visible spectrum. Nano.lett., 2009, 7, 2562-36.

[8] Lee, C.W.; Yang, S.H.; Hsu, C.S.; Meng, H.F. Improved efficiencies in organic light emitting diodes made with $\mathrm{CdSe} / \mathrm{ZnS}$ quantum dots and a semiconducting polymer. J. Nano sci. Nanotechnol,. 2009, 9(3), 2076-80

[9] Wang, Y.; Herron, N. Nanometer-sized semiconductor clusters: materials synthesis, quantum size effects, and photophysical properties. J. Phys. Chem., 1991, 95, 525-532.

[10] Herron, N.; Wang Y.; Eskert, H. Synthesis and characterization of surface-capped size-quantized cadmium sulfide clusters. Chemical control of cluster size. J. Am. Chem. Soc., 1990, 112, 1322-26.
[11] Cullity, B. Elements of X-Ray diffraction, II Ed. Addison-Wesley, Redding. 1978.

[12] Kayanuma, Y. Quantum Size effects of interacting electrons and holes in semiconductor microcrystals with spherical shape. Phys. Rev. B., 1998, 38, 9797-9805.

[13] Wilson, J.; Hawkes, J.F.B. Optoelctronics: An introduction. II Ed. Prentice-Hall India Pvt.Ltd., 1999, 122-126.

[14] Kalinowski, J. Electroluminescence in organics. J. Phys. D: Appl. Phys., 1999, 32, R179-R250.

[15] Yang, H.; Holloway, P.H. Enhanced photoluminescence from CdS:Mn/ZnS core/shell quantum dots. Appl. Phys. Lett., 2003, 82(12), 1965-1967.

Received: August 07, 2011

Revised: August 26, 2011

Accepted: October 04, 2011

(C) Vishwakarma et al.; Licensee Bentham Open.

This is an open access article licensed under the terms of the Creative Commons Attribution Non-Commercial License (http://creativecommons.org/licenses/by-nc/3.0/) which permits unrestricted, non-commercial use, distribution and reproduction in any medium, provided the work is properly cited. 